\title{
An improved method for mobility prediction using a Markov model and density estimation
}

\author{
Leonhard Menz*, University of Exeter; Roland Herberth*, Karlsruhe Institute of Technology; \\ Chunbo Luo, University of Exeter; Frank Gauterin, Karlsruhe Institute of Technology; \\ Ansgar Gerlicher, Stuttgart Media University; Qi Wang, University of the West of Scotland \\ *These authors contributed equally to this project and should be considered co-first authors.
}

\begin{abstract}
The prediction of an individual's future locations is a significant part of scientific researches. While a variety of solutions have been investigated for the prediction of future locations, predicting departure and arrival times at predicted locations is a task with higher complexity and less attention. While the challenges of combining spatial and temporal information have been stated in various works, the proposed solutions lack accuracy and robustness. This paper proposes a simple yet effective way to predict not only an individual's future location, but also most probable departure and arrival times as well as the most probable route from origin to destination.
\end{abstract}

Index Terms-Mobility behaviour, mobility prediction, markov model, probability density function

\section{INTRODUCTION}

Knowledge about individual mobility behaviour is useful for many applications such as urban planning or location-based services [1]. Therefore a number of recent research projects investigated different methods for human mobility prediction. The availability of large quantities of mobility data that has been collected with help of smart mobile devices, supported research projects in this field [2] [3]. Many of the conducted research projects focused on predicting an individual's future location, answering the question (Q1): "Where will the individual be at time $\mathrm{xx}$ :xx of day $\mathrm{y}$ ?"

However the question $(\mathrm{Q} 2)$ : "At what time is the individual leaving location A to arrive location B?" often remains unanswered. The complexity behind the answer for this question can be illustrated by the results of previously mentioned studies. It has been shown and will be confirmed by results of this paper that the majority of individuals move between few significant points of interest (POI). Knowing an individual's most significant places reduces the number of probable answers to the first question (Q1) drastically. The same cannot be said about departure times. Depending on the temporal resolution, statements about departure times are either inaccurate or not reasonable due to virtually infinite possible answers. Burbey [4] pointed out first that it is a different task and more complex to predict when an individual is at a specific location than where someone will be at a specific time. Baumann [1] showed that mobility traces are more predictable than residence and arrival times.

A key challenge in predicting detailed mobility behaviour is the combination of spatial and temporal data. Song et al. presented that a significant share of information lies within the temporal order of a visiting pattern [2]. Markov chains (MC) of first and second order are simple and effective tools for location predictions. Some projects based on Markov models (MM) achieved the accuracy of $>90 \%$ on location prediction. $\mathrm{MC}$ of first order are characterized by that the current state is solely dependent on the previous state. The so called "Mobility Markov Chains" [5] are adequate to predict locations but struggle when used to combine spatial and temporal data. Nevertheless a number of projects [1] [6] [7] [8] combined MC with temporal information in the form of time stamps. Inherent in the system the efficiency of MC suffer from temporal information (sparse data) and disadvantages outweigh advantages when high temporal granularity is desired. Also lots of historical data is needed for a system in order to be able to make reasonable predictions in terms of departure and arrival times. This leads to the following conclusions: Temporal information is often only used to improve location prediction. Combining location and time prediction is a less investigated field of research. This results in a lack of temporal accuracy. Presented solutions, especially MC, are generally independent of temporal information. Recent work like [1] [6] [7] [8] overcome this drawback by combining temporal and spatial information into the MM. However high temporal resolutions (e.g. $1 \mathrm{~min}$ ) are not suitable for MM.

In this paper we propose a framework to achieve high prediction rates with the help of a MM based transition matrix but in combination with high granularity in terms of departure and arrival times. By decoupling the prediction of "where" somebody is travelling to and "when" the individual is going to travel, we avoid the previously described drawbacks of combining spatial and temporal data in "Mobility Markov Models". The proposal will introduce a function that predicts mobility behaviour in a sequence of applications that have been proven to be effective for their specific fields. While future locations will be predicted by a MM inspired transition matrix of first order, departure and arrival periods are predicted with help of a probability density function (PDF).

The focus of this paper is on mobility that is usually performed with some kind of transportation vehicle, meaning that the covered distances are beyond average walking distances. This excludes walks within buildings that might sum up to significant length when combined. For instance, a person might "travel" several kilometres per day by visiting colleagues on different levels of a business building. In order to demonstrate the system's applicability, data will be used that has been collected from real persons. It will be demonstrated 
that the system is able to learn and predict trips that show a form of regularity. Compared with previous solutions the proposed scheme is capable of giving more precise predictions about the departure and arrival times as well as the route that is going to be taken. The proposed framework is simple and easy to calculate yet effective and accurate.

The structure of this paper continues as follows: Section II provides an overview of the state-of-the-art in mobility predictions; Section III introduces the proposed framework including the system model; Section IV analyses the performance using exemplary profiles; Section V discusses the implementation issues, the limitations of the model and future work; Section VI concludes this paper.

\section{RELATED WORK}

Recent results of research show various notable types of mobility prediction algorithms. Most can be categorized into information theoretic approaches like MM [9] [10] and compression algorithms (LZ family) [11] or machine learning methods like Bayesian networks, neural networks and pattern mining techniques [12]. Furthermore several improvements were presented over the years for these approaches. Nevertheless most of them only take spatial information into account while other information of trajectory especially temporal aspects are missing. MM and compression algorithms (LZ family) are common approaches because of their proportionally low complexity and resource needs. Markov based models are simple yet effective tools for mobility predictions. They have been successfully applied for predicting someone's next location. Depending on various factors such as randomness in behaviour and the data collection method prediction rates of $33-91 \%$ accuracy were reached [4].

A characteristic property of MC of first order is that the current state is only dependent on the previous state. Meaning that in case of mobility the current location is only dependent on the previous location. The key question of: "What is the most useful order of a MM for mobility prediction?" has been answered differently. The highest prediction accuracy for location prediction was achieved with a first order MM in [4]. To limit the models overhead and due to insignificant increase in accuracy of higher order models in other projects [13] the model proposed in this paper is based on a MM of first order.

Predictability in general has been examined from an information theoretic standpoint [11] [14] [15]. Therefore previous works calculated mobility data's entropy to determine their predictability. Their results confirm that "a significant proportion of information is encoded in the temporal order of visitation patterns" [4].

Recent works [6] [7] combined temporal and spatial information in a MM naming it Time-Based Markov (TBM). On one hand the results of [6] show a small increase of prediction accuracy from $38.2 \%$ to $39.2 \%$ when time information is included in the prediction process. On the other hand their time range of four hours (for which their prediction was most accurate) is rather inaccurate for departure and arrival time prediction. Cheng et al. improved the prediction accuracy with the consideration of time information by $6 \%$ compared with the original Markov algorithms [7]. In this case the selected time period amounts to one hour, meaning every day is divided into 24 time intervals. In fact for both TBM temporal information is only used to improve performance for location prediction. A lack of predicting when someone arrives still remains. By numbers [4] achieved the highest accuracies of $77 \%$ to $91 \%$ with a time tolerance of 20 minutes. While this can be seen as fairly accurate, it should be avoided to compare these values directly. As previously mentioned, the prediction performance is very much dependent on the used data sets. Also the performance measurements vary and make a direct comparison difficult.

\section{Methodology}

By decoupling temporal and location predictions we avoid previously mentioned drawbacks. Our approach does not only aim to forecast a user's next location, but also to predict departure and arrival times with high accuracy. This is done without loss of correlation between time and location. Unlike previous solutions temporal information will not be included directly into the MM. Instead a Probability Density Function (PDF) is used for temporal predictions. This means MM are exclusively used to predict a user's next location (where) while the PDF will be used to estimate departure times from the current location (when). Details of this approach will be presented and discussed in the following section.

\section{A. A Markov Model for mobility prediction}

$\mathrm{MM}$ are useful for modelling stochastic processes and can be used to specify systems with random state changes. They are described by a finite (or infinite) set of states $S=\left[s_{1}, \ldots, s_{n}\right]$ which defines the state space. Changeovers between those states depend on their transition probabilities which are defined in a set of transitions $T=\left[t_{1,1}, \ldots, t_{n, n}\right]$. Accordingly the transition probability from state $t_{1}$ to state $t_{2}$ is defined as $p_{12}$. The set of transition probabilities is represented as a transition matrix of the dimension $n \times n$ where $n$ is the number of states. In terms of analysing users' mobility behaviour, states represent (visited) POIs whereas the transition probabilities depend on the quantity of (driven) trips among POIs.

While high prediction accuracy rates prove that $\mathrm{MM}$ are applicable for mobility prediction, its characteristics stand in conflict with some human mobility behaviour. This can be illustrated by a simple example: An often observable behaviour of a working person is the departure from home to work in the morning followed by a return to home in the afternoon. According to the logic of a simple MC, the probability for the transition "Home $\rightarrow$ Work" and "Work $\rightarrow$ Home" increases with every time in which the person follows this behaviour. From a statistical point of view, the MM would answer the question: "Where is the person next, given he or she is at home?" most of the time correctly with "Work". However, for real world data there are some disadvantages in this logic. With increasing number of transitions "Home $\rightarrow$ Work", "Work $\rightarrow$ Home", this behaviour will outweigh any other transition for as many times as this behaviour exceeds the next most probable 
transition. The criticality of this feature becomes obvious when the number of "significant" locations is $>2$.

Presumably in many cases the assumption that the next place that is going to be visited is only dependent on the current location is false. Since a basic MC does not consider temporal information, the prediction for a Sunday would always be the same as for a Monday or any other regular working day. A simple but effective way to overcome this issue is to make a distinction between different days. This can be done by separating working days from weekends and holidays, Mondays from Tuesdays, and so on. Differentiating between days increases the model's complexity as well as the requirements on the collected data. However, the increased requirements have been justified by observations of previous researches and common sense which observed day-of-weekspecific repetitive behaviour in human mobility [2].

Hence in our application we make a distinction between days of the week. We use a modified approach of [16], as we separate time in day specific bins, meaning that for the prediction of the mobility behaviour of a Monday, historical data of all Mondays is taken only. The same applies for Tuesdays and so on. The logical disadvantage of this approach is that for a prediction that is, for instance, based on four days, data of one month is required (cold start problem).

\section{B. Probability Density Function for departure prediction}

Predicting someone's next location is an easier task than making a reasonable assumption about the corresponding departure time. This is because there are usually more temporalpredictions possible than feasible location-predictions. Due to sporadic human behaviour and unexpected external circumstances, such as traffic jams or getting a phone call, the prediction of arrival and departure times is always subjected to uncertainties. Even huge quantities of historical data cannot provide $100 \%$ certainty.

To increase the mobility prediction's quality we aim to determine the most probable departure time of an individual. Route and day specific departure times are therefore treated as a random variable that underlies an unknown probability function. More specifically we are searching the time of the day in which the relative likelihood for departing to a destination, that has been selected from a MM, is the highest. We therefore employ a density estimation on day and trip specific departure times. Based on [18] a density estimator $\hat{f}(x)$ is defined as

$$
\hat{f}_{n}(x)=\frac{1}{n b} \sum_{i=1}^{n} K\left(\frac{x-x_{i}}{b}\right)
$$

where $K$ is a kernel function, $n$ number of samples and $b>0$ is the bandwidth. We are less interested in the actual underlying probability function but more in a reliable indication of a probable departure time which will be described in the following section.

\section{Model description}

Other than previous proposals we separate the task of prediction where somebody is heading to and when somebody is leaving. While a MM based transition matrix is used to determine an individual's probable next destination, the corresponding departure time is calculated after the most probable destination has been determined. The major benefit of this process is that the performance of the MM is not influenced by the inclusion of temporal information.

\section{Model implementation}

As MM showed good performance in previous works, we implement a MM of first order. Based on [17] we describe our model with the 'Probability Theory'. Let $X$ be a set of POIs that represent Origins and $Y$ be a set of POIs that represent Destinations. Every POI can be both an origin and a destination. $X$ can take any value of $x_{i}$ where $i=1, \ldots \mathrm{M}$, and $Y$ can take any value of $y_{j}$ where $j=1, \ldots, \mathrm{L}$, forming a $\mathrm{L} \times \mathrm{M}$ matrix. Let $N$ be an individual's POI sequence where the sequences' last item is the individual's current location $x=x_{c l}$. If a specific location from a set of locations is denoted as $X=x_{i}$ and similarly a specific destination from a set of destinations is denoted as $Y=y_{j}$ the probability $p$ for the event $\left(X=x_{i}, Y=y_{j}\right)$ is called joint probability and calculated with

$$
p\left(X=x_{i}, Y=y_{j}\right)=\frac{n_{i j}}{N}
$$

where $n_{i j}$ is the cell $i, j$ as fraction of all cells of matrix $\mathrm{L} \times \mathrm{M}$.

For now, suppose that we are interested in finding the probabilities for transitioning to known destinations, given that a POI sequence is used to count the number of transitions between $X$ and $Y$. Since the origin and Current Location $(\mathrm{cl})$ is known $\left(x=x_{c l}\right)$, the conditional probability for known destinations is calculated with

$$
p\left(Y=y_{j} \mid X=x_{c l}\right)=\frac{n_{c l, j}}{N_{Y}}
$$

where $n_{c l, j}$ is the number of transitions from $x_{c l}$ to $y_{j}$ and $N_{Y}(c l)=\sum_{j=1}^{L} n_{c l, j}$. Note that when a user is visiting a place that has not been visited before, the conditional probability for all destinations is 0 and no prediction can be made. However, given that the individual is in a known location, the model is able to provide transition probabilities to all known places that have been reached from the current location. We grade all possible destinations in a descending order and declare the first entry as the most probable destination. From our database we request all trips that lead from the current location to the predicted destination. To cope with variations in daily habits we select day specific departure times of the requested trips and form a univariate density estimation to determine the most probable departure time.

We assume a close to normal distributed probability for departing around a typical departure time for predicted trips. Therefore we employ a normal (Gaussian) kernel

$$
K(x)=\Phi(x)
$$

for a density estimation with a variable bandwidth

$$
b=\left(\frac{4 \hat{\sigma}^{5}}{3 n}\right)^{\frac{1}{5}}
$$


that minimizes the mean squared error (Silverman's rule [18]).

To limit computation time for later steps we make a point prediction rather than an interval prediction and choose the maximum likelihood that is the highest peak of the density function to determine the most probable departure time. If the point of time that marks the most probable departure (time of day) is in the past of the current daytime, we repeat the departure time request for the second most probable destination, provided by the MM. We repeat this step for the third most probable destination if the most probable departure time for the second most probable destination is also in the current day's past. If the most probable departure time for the third most probable destination is also in the current day's past, we assume that no further trip is taken at this specific day.

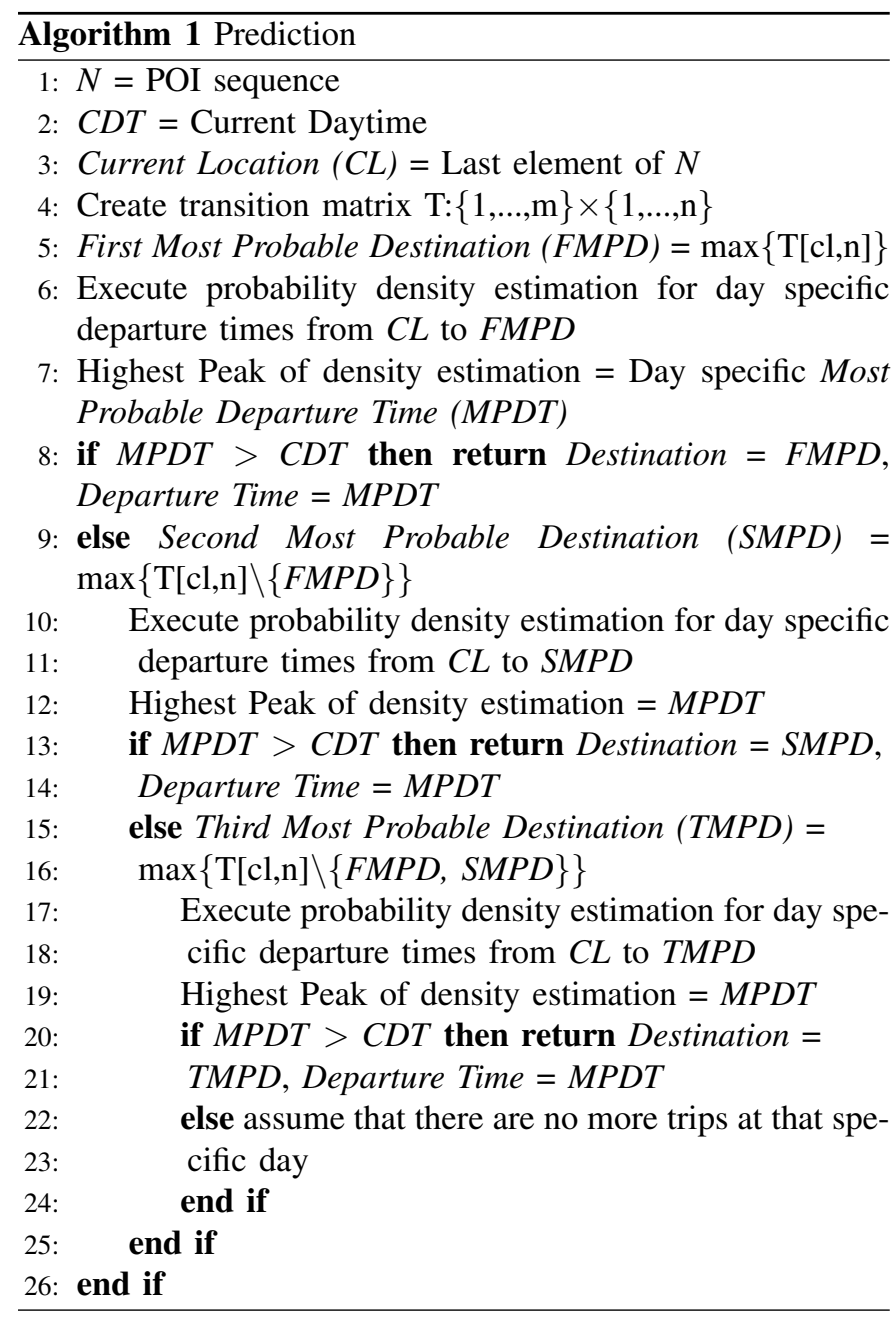

If we predicted a trip that, according to the density function, is yet to come, we use the median trip duration of all trips that comply with the corresponding origin destination couple to provide a probable arrival time at the predicted destination. Being the most probable scenario, the predicted arrival time together with the predicted destination is the new starting point for the next trip prediction. Doing so provides us a system that is able to make any number of predictions with however decreasing accuracy (probability) for long time predictions. In this case the system acts as if the vehicle is located in the predicted POI at the predicted arrival time and requests the next most probable destinations.

Figure 1 illustrates specific departure events to the most probable destination in form of a histogram. The red line shows the kernel smoothing as function of departure times. The area below the curve can be used to determine the probability of departure within time specific periods. We use the highest peak of the smoothing function for the prediction of departure time.

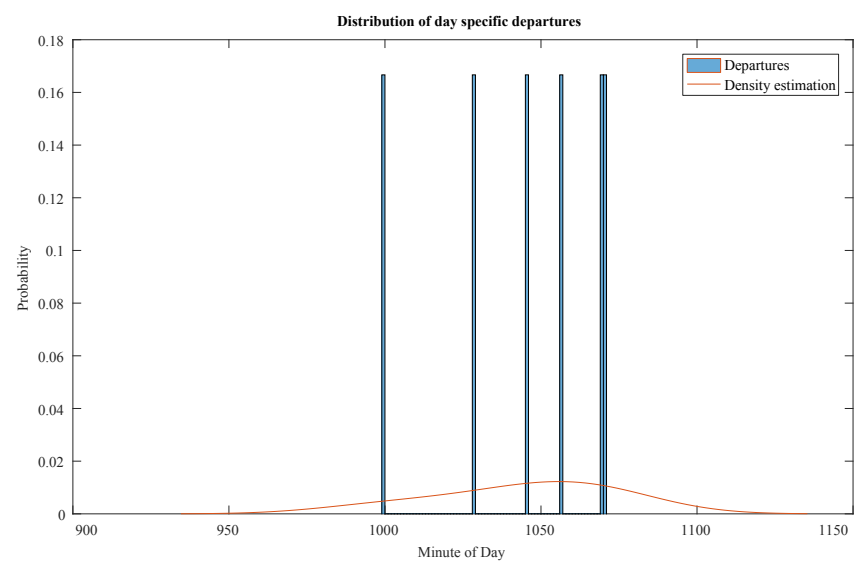

Fig. 1. Density estimation with Kernel smoothing function consisting of six departure samples showing a peak at 1055. The departure event therefore corresponds to $17: 35 \mathrm{pm}$.

\section{E. Parametrization}

Figure 2 displays the distribution of visitations per POI. The number of relevant POIs for all observed users ranges between 3 to 6 . To avoid overfitting we only consider the first three

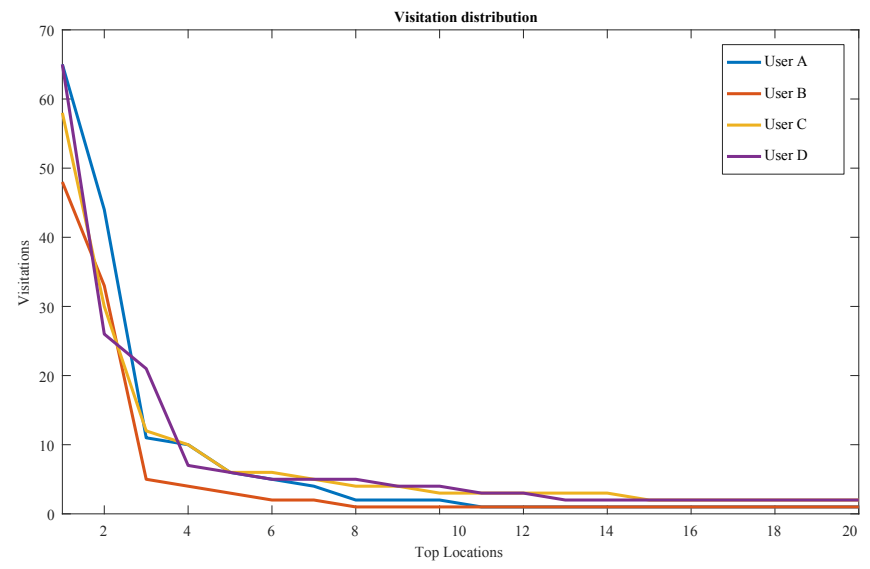

Fig. 2. Distribution of visitations per POI by all users. The graphs shows that the number of top visited locations ranges between 3 to 6 for all users.

location based most probable destinations for our prediction. We use three as threshold as it provides a reasonable trade off between significant locations and locations that have been visited in insignificant proportion, as displayed in Figure 2. This observation is supported by [4] which achieved best prediction rates when not more than three locations have been visited in one timeslot. 


\section{EXPERIMENTAL RESULTS}

\section{A. Data pre-processing}

The MM as well as the density estimation requires to preprocess trajectory data. For the proposed model it is necessary to identify POI's as well as repetitive driven trips and their temporal assignment. As our raw data consists of GPS data in conjunction with unixtime, we conduct two different steps to pre-process the collected data.

GPS-Based Points of Interest Identification - To identify POI's we use both the first and the last GPS position of a trip and define them as place points. After a new trip is finished, it is checked if its start and end place points are within a radius of 500 metres with a previously recorded place point or a previously formed POI. If a previously recorded place point is in range, the mean of new and old place points form a now POI. If a previously formed POI is in range, the new place point is assigned to the POI. The radius was determined empirically as it provided the most accurate results for our test data set. Once a POI is formed, a POI-ID is assigned.

GPS-Based Trip ID Identification - To support the application of a density estimation for departure times we are interested in the occurrences of similar (departure) events. We define these events not only by departing from A to B but also by the route that has been driven from origin to destination. This requires to compare and identify similar trips. This is realized by calculating the average of the straight line distances between every GPS point and its closest segment of the opposing route. If the average distance is equal or smaller than 100 metres, the compared trips are considered to be equal and a dedicated trip-ID is assigned. The distance of 100 metres has also been determined empirically as it provided the best results.

The assignment of POI- and trip-IDs allows to describe an individual's mobility behaviour as sequence of POIs and trips which is the basis for the creation of a Markov based transition matrix.

\section{B. Profile regularity}

Prediction accuracy strongly relies on the regularity of a user's profile. Previous work showed that the entropy $S$ (information theory) can be used as an indicator to measure this regularity. Therefore the degree of entropy sets a theoretical limit in predictability, meaning that for individuals with a lower mobility entropy the theoretic predictability is higher and vice versa. Recent literature show several variations to determine entropy. Various sources introduced and referred a methodology based on the Lempel-Ziv data compression algorithm [2] [13] [19]. However latest results in [19] showed that entropy strongly depents on cluster radius $(\Delta s)$ of POIs and temporal resolution $(\Delta t)$ of the trajectory data. $\mathrm{Xu}$ et al. [20] showed an alternative approach called "refined composite multiscale entropy" (RCMSE) which is an improved approach of the multiscale entropy. Finally Qin et al. calculate their entropy based on a day specific comparison [21]. Our available data set allows a similar calculation of the entropy $\mathrm{S}$ :

$$
S=\frac{\sum_{j=1}^{N} \sum_{i \in I}\left(\frac{l_{i j}}{D_{t o t}}\right) * \log \left(\frac{l_{i j}}{D_{t o t}}\right)}{T_{t o t}}
$$

where the number of time slots in one day is $T_{\text {tot }}$, the number of days is $D_{t o t}, I$ is the set of all visited locations and $l_{i j}$ is the number of times location $i$ dominates time slot $j$.

\section{Performance analysis}

We test the first implementation of the algorithm on a real world dataset. Figure 3 illustrates recorded data for five consecutive weeks for user A. Note that we can differentiate between trips however for easier recognizability all trips are coloured dark blue. Different colours show presence at different locations. The $\mathrm{x}$-axis displays Time of day, the $\mathrm{y}$ axis shows Days. Rows from the top to the bottom are in consecutive order.

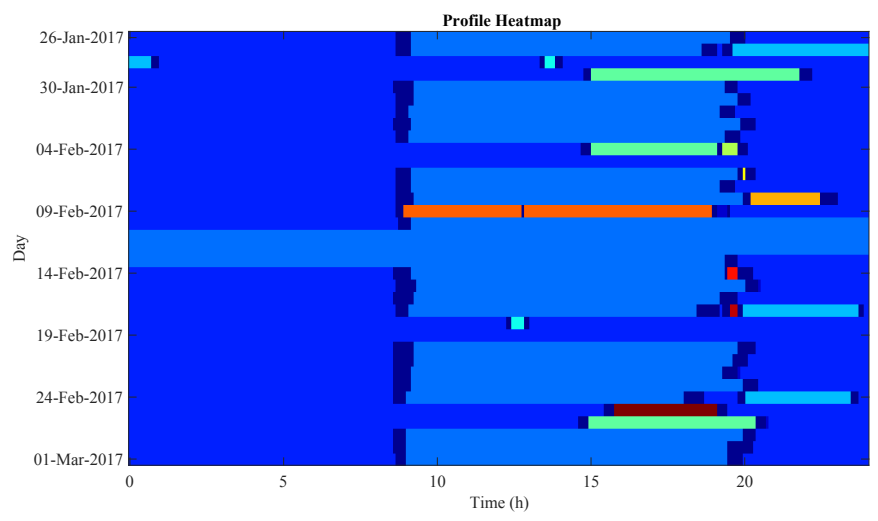

Fig. 3. Data visualisation of the recorded profile from user A, showing high regularity of moving between home and work location on weekdays. Departures around 9:00 am are highly consistent.

We use eight weeks of recorded data for the prediction of one week. Figure 4 shows the system response.

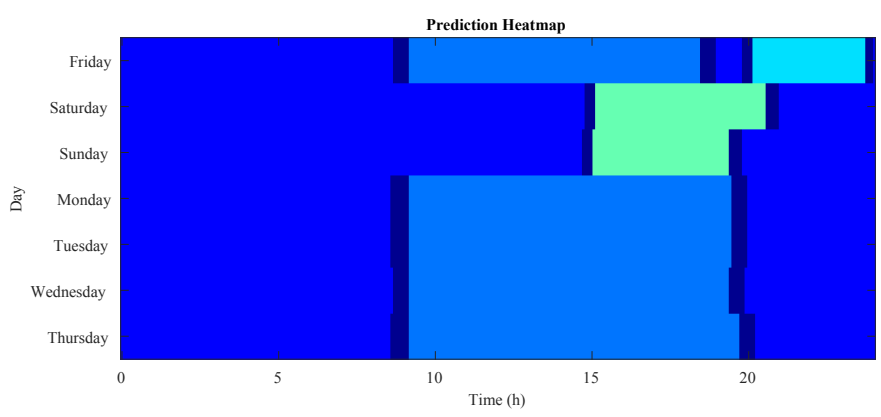

Fig. 4. Data visualisation of the predicted profile from user A. All frequently visited places on weekdays and weekend appear in the system's response.

To demonstrate the algorithm's performance we use four different available datasets that were collected by test users.

Table I displays the predictions accuracy of four different users and the corresponding profile entropy. The overall accuracy is calculated with

$$
\text { Accuracy }=\frac{\text { correctPredictions }}{\text { allPredictions }} \text {. }
$$

The average profile entropy is an indicator for regularity. While an entropy of 0 would indicate that the profile is completely 


\begin{tabular}{lllll} 
Day of Week & User A & User B & User C & User D \\
\hline Monday & $85.29 \%$ & $75.52 \%$ & $72.61 \%$ & $74.08 \%$ \\
Tuesday & $93.67 \%$ & $76.38 \%$ & $71.94 \%$ & $70.12 \%$ \\
Wednesday & $81.47 \%$ & $83.32 \%$ & $61.58 \%$ & $70.94 \%$ \\
Thursday & $84.79 \%$ & $75.31 \%$ & $64.74 \%$ & $69.00 \%$ \\
Friday & $83.81 \%$ & $65.30 \%$ & $63.14 \%$ & $67.24 \%$ \\
Saturday & $62.23 \%$ & $58.56 \%$ & $80.36 \%$ & $61.79 \%$ \\
Sunday & $67.33 \%$ & $68.99 \%$ & $75.93 \%$ & $69.02 \%$ \\
\hline Average & $79.80 \%$ & $71.91 \%$ & $70.04 \%$ & $68.88 \%$ \\
\hline Entropy & 0.68 & 0.76 & 1.12 & 0.97 \\
& TABLE I \\
\end{tabular}

PERFORMANCE ON TEST DATA

\begin{tabular}{lllll} 
Day of Week & User A & User B & User C & User D \\
\hline Monday & 22 & 19 & 17 & 30 \\
Tuesday & 24 & 32 & 32 & 22 \\
Wednesday & 18 & 28 & 13 & 21 \\
Thursday & 17 & 51 & 1 & 24 \\
Friday & 18 & 26 & 34 & 49 \\
Saturday & 13 & - & 114 & - \\
Sunday & 33 & - & - & - \\
\hline Average & 21 & 31 & 35 & 29
\end{tabular}

MAE IN MINUTES ON CORRECTLY PREDICTED TRIPS

predictable, high entropy indicates little predictability. Table II displays the mean absolute error (MAE) of departure time prediction in minutes for correctly predicted trips.

\section{Discussion}

A major source of error is the quality of GPS data. For persistent data input all vehicles used for data collection were equipped with GPS-dongles that use the vehicle's onboard diagnostics port for power supply. The used hardware is more prone to connection abortion than systems that use the vehicle's external GPS antenna as the dongles are connected inside of the vehicle's passenger cabin. This led to situations in which not the entire trip was recorded due to missing GPS connections. Missing parts of trajectories led to situation in which the trip start and end could not be correctly assigned to POIs what consequently led to wrong departure and arrival time assignments. We expect performance improvements with better data collection hardware. The proposed framework separates days on weekly cycles. Inherent in the system the model does not consider patterns that lie outside of weekly intervals. Hence behaviour that is observable every 8th day or every other week is being neglected.

\section{CONCLUSion AND Future Work}

This paper introduces a new framework that combines the application of MM for location prediction and PDF for departure time prediction. Since spatial and temporal predictions are different tasks, the model separates them but without loss of correlation between POIs and time. Naturally, the model achieves the highest prediction accuracy for mobility behaviour with high regularity, indicated by a low entropy.
Results show good performance for weekly patterns however there are some points which can be addressed in order to improve the algorithm's performance. For instance, the introduction of a higher level, that separates time into weekly cycles could cope the previously described limitations concerning daily cycles. Also further improvements could include the acquisition of multiple data sources in order to ensure a maximum coverage of mobility data so that missing data can be compensated by other sources, such as user specific calendar entries. Integration of further sources will be part of improvements for future versions of the proposed model.

\section{REFERENCES}

[1] Paul Baumann, "Human mobility and aplication usage prediction algortihms fof mobile devices," Ph.D. dissertation, TU Dresden, Dresden.

[2] Chaoming Song, Zehui Qu, Nicholas Blumm, and Albert-László Barabási, "Limits of predictability in human mobility," 19 February 2010.

[3] Myungjun Choi, Yonghyun Ro, and Han Lee, "Human mobility pattern prediction algorithm using mobile device location and time data," 2013.

[4] I. Burbey, "Predicting future locations and arrival times of individuals," Dissertation, Virginia Polytechnic Institute and State University, Blacksburg, Virginia, 26 April, 2011.

[5] Sebastien Gambs, Marc-Olivier Killijian, and Miguel Nunez del Prado Cortez, "Show me how you move and i will tell you who you are," Transactions On Data Privacy4 (2011) 103-126, 2011.

[6] Haiyang He, Yuanyuan Qiao, Sheng Gao, Jie Yang, and Jun Guo, "Prediction of user mobility pattern on a network traffic analysis platform," ACM, 2015.

[7] Yihang Cheng, Yuanyuan Qiao, and Jie Yang, "An improved markov method for prediction of user mobility."

[8] Sebastien Gambs and Marc Olivier Killijian, "Towards temporal mobility markov chains," 1st International Workshop on Dynamicity Collocated with OPODIS 2011, 2011.

[9] Daniel Ashbrook and Thad Starner, "Using gps to learn significant locations and predict movement across multiple users," In: Proceedings of the 6th IEEE International Symposium on Wearable Computers, vol. 2002.

[10] Dimitrios Katsaros and Yannis Manolopoulos, "Prediction in wireless networks by markov chains," IEEE Wireless Communications, no. Volume: 16, Issue: 2, 2009.

[11] Karthik Gopalratnam and Diane J. Cook, "Active lezi: An incremental parsing algorithm for sequential prediction," American Association for Artificial Intelligence, vol. 2004.

[12] Sungjun Lee, Junseok Lim, Jonghun Park and Kwanho Kim, "Next place prediction based on spatiotemporal pattern mining of mobile device logs," MDPI, 23 January 2016.

[13] Xin Lu, Erik Wetter, Nita Bharti, Andrew J. Tatem, and Linus Bengtsson, "Approaching the limit of predictability in human mobility," SCIENTIFIC REPORTS 3 : 2923, 2013.

[14] Alicia Rodriguez-Carrion, Carlos Garcia-Rubio, Celeste Campo, Alberto Cortes-Martin, Estrella Garcia-Lozano, and Patricia Noriega-Vivas, "Study of lz-based location prediction and its application to transportation recommender systems," sensors, no. 1424-8220, 4 June 2012.

[15] Amiya Bhattacharya and Sajal K. Das, "Lezi-update: An informationtheoretic approach to track mobile users in pcs networks," In Mobile Computing and Networking, pp. 1-12, 1999.

[16] Reid Simmons, Brett Browning, Yilu Zhang, and Varsha Sadekar, "Learning to predict driver route and destination intent," IEEE Intelligent Transportation Systems Conference, 2006.

[17] C. M. Bishop, Pattern Recognition and Machine Learning. Springer Science+Business Media, 2006.

[18] B.W. Silverman, Density Estimation For Statistics and Data Analysis: Statistics and Applied Probability, 1986.

[19] Edin Lind Ikanovic and Anders Mollgaard, "An alternative approach to the limits of predictability in human mobility," EPJ Data Science, 2017.

[20] Tao Xu, Xianrui Xu, Yujie Hu, and Xiang Li, "An entropy-based approach for evaluating travel time predictability based on vehicle trajectory data," Entropy, vol. 11 April 2017.

[21] Shao-Meng Qin, Hannu Verkasalo, Mikael Mohtaschemi, Tuomo Hartonen, and Mikko Alava, "Patterns, entropy, and predictability of human mobility and life," PLOS one, December 26, 2012. 